\title{
Preliminary investigation of an ultrasound method for estimating pressure changes in deep-positioned vessels.
}

Olesen, Jacob Bjerring; Villagómez Hoyos, Carlos Armando; Traberg, Marie Sand; Chee, Adrian J. Y. ; Yiu, Billy Y. S.; Ho, Chung Kit; Yu, Alfred C. H.; Jensen, Jørgen Arendt

\section{Published in:}

Proceedings of SPIE

Link to article, DOI:

$10.1117 / 12.2214974$

Publication date:

2016

Document Version

Peer reviewed version

Link back to DTU Orbit

Citation (APA):

Olesen, J. B., Villagómez Hoyos, C. A., Traberg, M. S., Chee, A. J. Y., Yiu, B. Y. S., Ho, C. K., Yu, A. C. H., \& Jensen, J. A. (2016). Preliminary investigation of an ultrasound method for estimating pressure changes in deeppositioned vessels. In N. Duric, \& B. Heyde (Eds.), Proceedings of SPIE (Vol. 9790). [97900J] SPIE -

International Society for Optical Engineering. https://doi.org/10.1117/12.2214974

\section{General rights}

Copyright and moral rights for the publications made accessible in the public portal are retained by the authors and/or other copyright owners and it is a condition of accessing publications that users recognise and abide by the legal requirements associated with these rights.

- Users may download and print one copy of any publication from the public portal for the purpose of private study or research.

- You may not further distribute the material or use it for any profit-making activity or commercial gain

- You may freely distribute the URL identifying the publication in the public portal 


\title{
Preliminary investigation of an ultrasound method for estimating pressure changes in deep-positioned vessels
}

\author{
Jacob Bjerring Olesen ${ }^{a}$, Carlos Armando Villagomez-Hoyos ${ }^{a}$, Marie Sand $\operatorname{Traberg}^{a}$, \\ Adrian J. Y. Chee ${ }^{b}$, Billy Y. S. Yiu ${ }^{b}$, Chung Kit $\mathrm{Ho}^{b}$, Alfred C. H. Yu ${ }^{c}$, and \\ Jørgen Arendt Jensen ${ }^{a}$ \\ ${ }^{a}$ Center for Fast Ultrasound Imaging, Dept. of Elec. Eng., Bldg. 349, \\ Technical University of Denmark, DK-2800 Kgs. Lyngby, Denmark \\ ${ }^{b}$ Medical Engineering Program, University of Hong Kong, Pokfulam, Hong Kong \\ ${ }^{c}$ Department of Electrical and Computer Engineering, University of Waterloo, Waterloo, \\ Ontario, Canada.
}

\begin{abstract}
This paper presents a method for measuring pressure changes in deep-tissue vessels using vector velocity ultrasound data. The large penetration depth is ensured by acquiring data using a low frequency phased array transducer. Vascular pressure changes are then calculated from 2-D angle-independent vector velocity fields using a model based on the Navier-Stokes equations. Experimental scans are performed on a fabricated flow phantom having a constriction of $36 \%$ at a depth of $100 \mathrm{~mm}$. Scans are carried out using a phased array transducer connected to the experimental scanner, SARUS. 2-D fields of angle-independent vector velocities are acquired using directional synthetic aperture vector flow imaging. The obtained results are evaluated by comparison to a 3 -D numerical simulation model with equivalent geometry as the designed phantom. The study showed pressure drops across the constricted phantom varying from $-40 \mathrm{~Pa}$ to $15 \mathrm{~Pa}$ with a standard deviation of $32 \%$, and a bias of $25 \%$ found relative to the peak simulated pressure drop. This preliminary study shows that pressure can be estimated non-invasively to a depth that enables cardiac scans, and thereby, the possibility of detecting the pressure drops across the mitral valve.
\end{abstract}

Keywords: Medical ultrasound, pressure estimation, vector flow imaging, synthetic aperture

\section{INTRODUCTION}

Measuring pressure variations is used clinically as a diagnostic marker for the physiological state of a cardiovascular region. ${ }^{1}$ Current clinical procedures for assessing pressure changes uses invasive devices such as pressure sensing catheters. ${ }^{2,3}$ However, these devices suffer severe limitations as they are invasive and require the use of ionizing radiation for guidance and positioning. To overcome the concerns related to the use of pressure catheters, several non-invasive alternatives for determining the pressure changes has been introduced. ${ }^{4-8}$ Among these techniques are medical ultrasound, where the ability of detecting blood flow in 1-D and 2-D can be used for deriving changes in intravascular blood pressure. ${ }^{9-12}$ Two-dimensional flow estimators allow for a better understanding of flow patterns, making it possible to derive the vascular pressure through more general approaches, such as the Navier-Stokes equations. However, up until today, pressure estimation based on 2-D flow estimators has been restricted to linear array transducers, thus, limiting the field of view to superficial vessels. But new developments in the velocity estimation scheme has made it possible to obtain angle-independent velocity data using phased array transducers, allowing for the opportunity to perform scans on deep-positioned vessels. This potentially leads to a method that can detect pressure changes across heart valves without using invasive catheters, or being dependent on the insonification angle of the ultrasonic beam.

The paper presents the first phantom study of measuring pressure changes from vector velocity flow data acquired using synthetic aperture with a phased array ultrasound probe. The initially obtained results are evaluated against a finite element model with a flow domain identical to the examined phantom. The work is an extension of previous publications by the authors, where the presented technique was tested for linear array probes. $^{12,13}$

Send correspondence to J. B. Olesen, E-mail: jbjol@elektro.dtu.dk 


\section{METHOD}

The suggested method is based on the Navier-Stokes equations:

$$
\rho\left[\frac{\partial \vec{v}}{\partial t}+\vec{v} \cdot \nabla \vec{v}\right]=-\nabla p+\rho \vec{g}+\mu \nabla^{2} \vec{v}
$$

presuming the conservation of mass and linear momentum. Eq. 1 describes the development of a fluid's velocity field $\vec{v}(\vec{r}, t)=\left(v_{x}(t), v_{y}(t), v_{z}(t)\right)$ by relating the forces acting on an incompressible volume to its acceleration and density throughout time, $t$, and space, $\vec{r}$. The left-hand side sums the local time-dependent acceleration, $\frac{\partial \vec{v}}{\partial t}$, and convective fluid acceleration $\vec{v} \cdot \nabla \vec{v}$, where $\rho$ is the density of the fluid and $\nabla$ is the spatial differential operator $\left(\frac{\partial}{\partial x}, \frac{\partial}{\partial y}, \frac{\partial}{\partial z}\right)$. The right-hand side shows the surface and volume forces that are responsible for the acceleration of the fluid. The forces constitute; a pressure drop $-\nabla p$, a gravitational force $\vec{g}$, and a viscous drag caused by the viscosity of the fluid $\mu \nabla^{2} \vec{v}$, where $\nabla^{2} \vec{v}$ is the Laplacian of the velocity field. The gravitational term is usually neglected, as a patient undergoing an ultrasound scan is placed in a supine position, hence, the buoyancy force cancels out the gravitational force.

For most clinical applications, the effect of the viscous term in (1) can be omitted, as this has no significant influence on the net-force in comparison with the inertial forces. This is especially the case in larger vessels for which the area of the boundary layer is small compared with the flow region beyond this layer, where flow is said to behave as an inviscid fluid. ${ }^{14,15}$

The high precision and temporal frame-rate offered by synthetic aperture flow ${ }^{16,17}$ allows for tracking of particles, which intuitively can be visualized by a streamline representation. Rewriting (1) into a one-dimensional equation following a streamline, where the influences of gravity and viscosity are omitted, yields the Euler equation;

$$
\frac{\partial p}{\partial s}=-\rho\left[\frac{\partial v_{s}}{\partial t}+v_{s} \frac{\partial v_{s}}{\partial s}\right]
$$

where $v_{s}$ is the scalar product of $\vec{v}(\vec{r}, t)$ and the unit vectors $\left(\hat{\mathbf{e}}_{x}, \hat{\mathbf{e}}_{y}, \hat{\mathbf{e}}_{z}\right)$. Integrating (2) along the flow direction of the streamline gives the drop in pressure across the line,

$$
\Delta P(t)=\int_{0}^{L} \frac{\partial p}{\partial s} d s .
$$

A streamline representation can be achieved by tracking the particle movement within the scan plane insonified by the ultrasound probe. This study employs a velocity estimator, which yields the two-dimensional (2-D) inplane vector velocity field $\vec{v}=\left(v_{x}, v_{z}\right)$. The proposed method is, thus, developed assuming that the out-of-plane velocity $v_{y}$ is zero.

\section{EXPERIMENTAL SET-UP}

The proposed method is evaluated on a straight-tube phantom with a $36 \%$ constriction during steady flow conditions. A part of the model is shown in Fig. 1. The phantom is fabricated in polyvinyl alcohol (PVA) cryogel designed to mimic anthropomorphic properties in terms of tensile strength and attenuation. ${ }^{18}$ The fabricated phantom is connected to a flow system (CompuFlow 1000, Shelley Medical Imaging Technologies, Toronto, Canada) capable of generating customized flow waveforms. The study is performed using a constant waveform and a flow rate of $5 \mathrm{ml} / \mathrm{s}$, and a $3 \mathrm{MHz}$ phased array probe with 128 elements (Sound Technology, Analogic Ultrasound Group, State College, PA, USA). A two-cycle pulse is emitted at a pulse repetition frequency of $6.2 \mathrm{kHz}$ to a depth of $120 \mathrm{~mm}$, with an elevation focus at $80 \mathrm{~mm}$. Five low-resolution images are summed for each high resolution flow image producing an effective frame-rate of $1.03 \mathrm{kHz}$. The ultrasound data are processed as proposed in, ${ }^{19}$ using five emissions instead of three. Data are processed off-line in Matlab (The MathWorks Inc., Natick, MA, USA). 


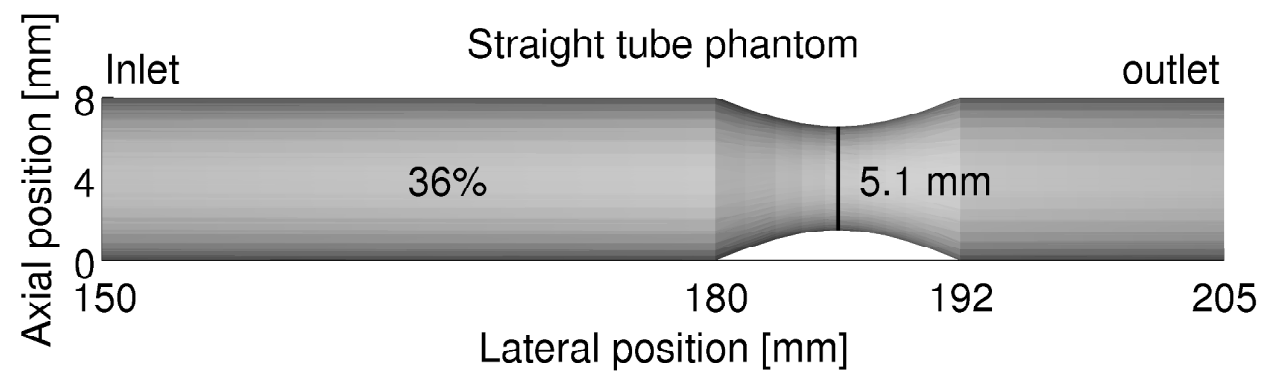

Figure 1. Fabricated flow model with a $36 \%$ constriction.

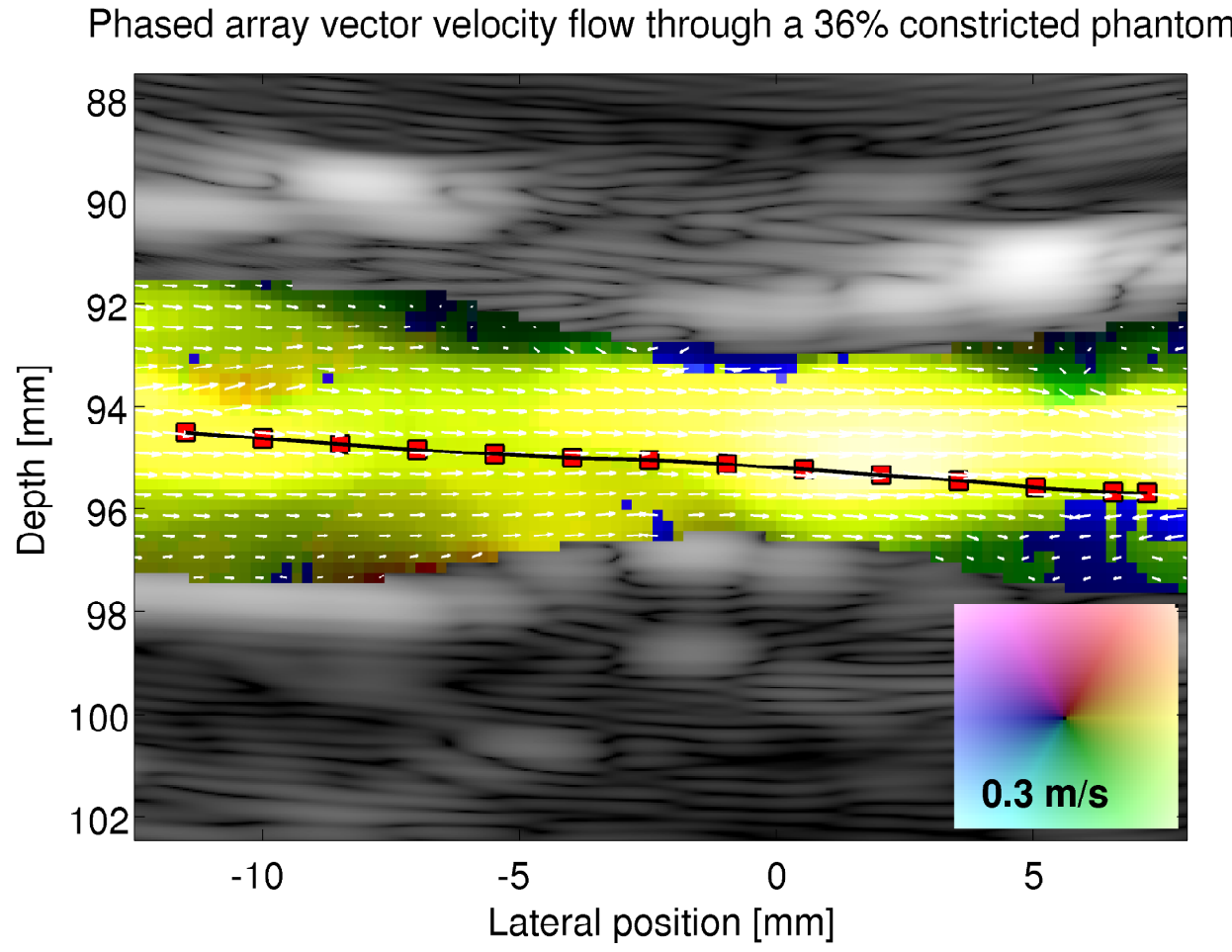

Figure 2. Vector velocity image at the constriction of the fabricated flow phantom. The image is captured during steady flow conditions. A black-dotted streamline passing through the center of the constriction is also displayed.

\section{FINITE ELEMENT MODEL}

The derived pressure gradients are validated against a finite element (FE) model made in Comsol (Comsol v5.1, Comsol AB, Stockholm, Sweden). The designed model holds the same geometry as the scanned phantom, thereby, ensuring identical flow domains between simulations and experimental models. Flow parameters in the FE model are set to mimic the flow conditions in the experimental set-up, with an input flow rate of $5 \mathrm{ml} / \mathrm{s}$, and a simulated viscosity and density of $4 \cdot 1 \cdot 10^{-3} \mathrm{~Pa} \cdot \mathrm{s}$ and $1,030 \mathrm{~kg} / \mathrm{m}^{3}$, respectively.

\section{RESULTS}

The method is tested for vector velocity flow data captured from the site of the $36 \%$ constriction. An example is displayed in Fig. 2. The image shows flow that accelerates toward the center of the constriction, producing a jet that then slowly decelerates as the lumen expands again. A black streamline following the vector field is also displayed. For the rest of the paper, changes in pressure are derived along this streamline. A bias of $-50 \%$ is observed in the measured flow data compared to the simulation model. ${ }^{19}$ This bias is used for compensating the velocity data, before inserting it in the pressure estimator. 


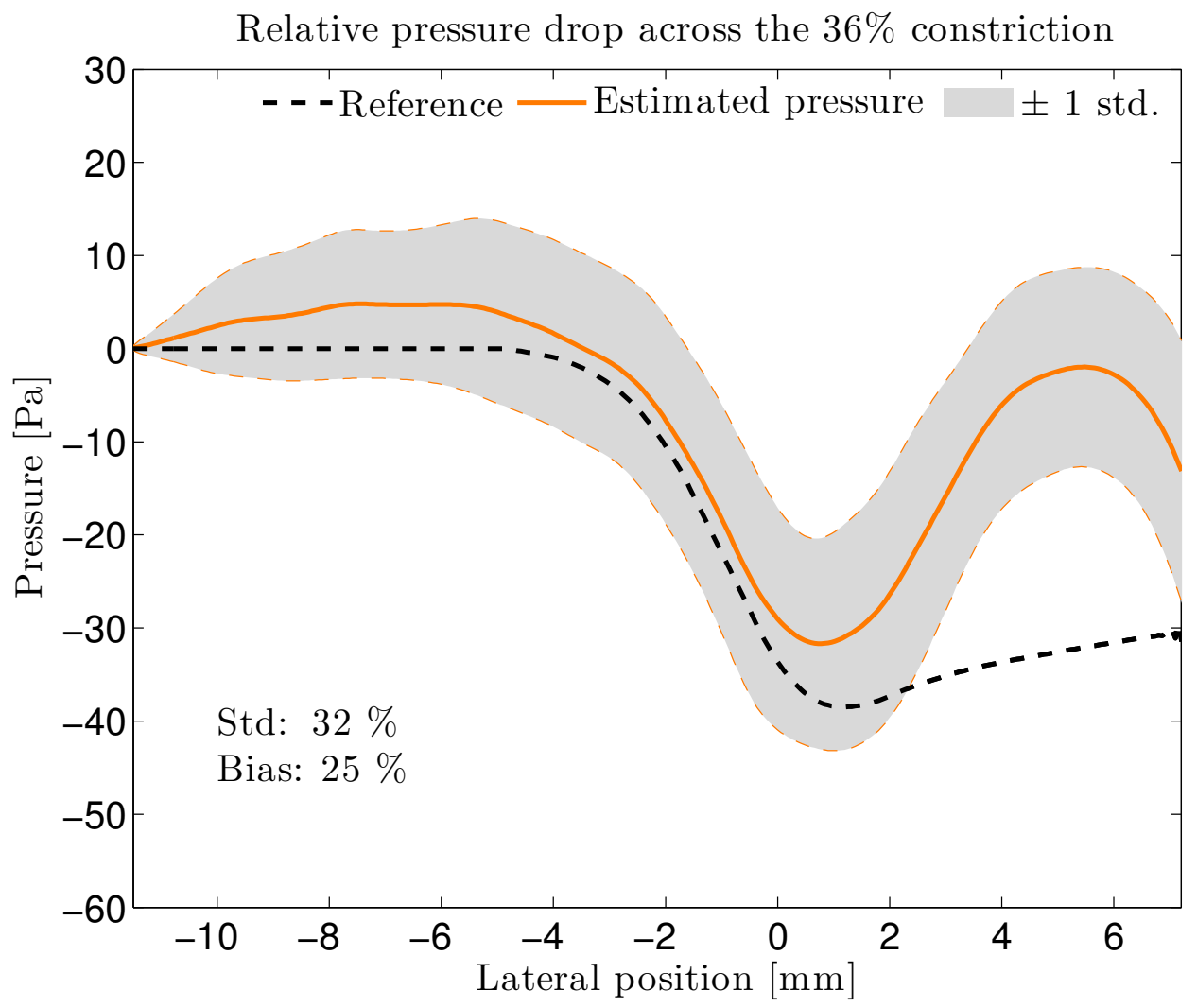

Figure 3. Constant flow: Estimated and simulated pressure drop through the center of a $36 \%$ constriction. The mean estimate is plotted together with \pm 1 standard deviation, and the output from the FE models.

For every position along the streamline, an individual pressure gradient is calculated before putted into (3). The individual gradients are found relative to its neighbouring estimates by employing polynomial filtering as introduced by Savitzky and Golay. ${ }^{20}$ However, as only spatial changes in velocity exist for steady flow, the temporal acceleration in (2) is neglected. The remaining spatial derivatives are calculated using a window size of five data points and a polynomial order of two. Then, calculating the cumulative sum of the local pressure changes along the direction of the flow, gives the relative drop in pressure that exist between the start of the streamline to any position along the line. The result of doing this for every measured velocity frame is plotted in Fig. 3. The figure shows a relative drop in pressure as the fluid moves through the constricted part of the phantom. The shaded zone indicates a region of one standard deviation normalized to the number of frames recorded, which in this case is 264 frames. The dotted dark curve in Fig. 3 is the result from the simulation model.

\section{DISCUSSION}

A non-invasive method for detecting pressure changes in a deep-positioned vessel using ultrasound vector velocity data has been presented. The study showed pressure changes across a $36 \%$ concentric constriction with a standard deviation of $32 \%$, and a bias of $25 \%$ compared to an FE simulation model. The bias changes dramatically as the streamline moves passed the center of the constriction. Whereas, a good match is seen prior to the constriction it suddenly changes for the worse after the narrowing. The reason for this is likely found in the fluid dynamic factors responsible for the drop in pressure and the employed method capability of detecting these. A pressure drop across an arterial stenosis is mainly govern by three factors: Friction seen in the fluid/wall-interaction or between adjacent layers of the fluid, pressure losses due to energy dissipation from turbulent flow features, and flow acceleration. The presented model derives pressure changes solely by measuring the effect from one of these factors, namely the pressure drop caused by convective flow. This explains the good correspondence seen prior 
to the constriction, as flow here behaves laminar, thus, changes in pressure are primarily caused by convective acceleration as also captured by the method. However, moving passed the constriction the flow starts to diverge and this leads to more turbulent flow features e.g. eddies and localized turbulence. Pressure changes caused by such features are not captured by the presented method, and that produces a greater bias between the estimated results and the reference model.

No previous studies on the subject have estimated pressure gradients from 2-D angle-independent vector velocity data obtained using a phased array. This however, allows for scanning vessels that are located deep in the tissue, as for instance the aorta or across the valves of the heart. The proposed method, further, offers the advantage of including temporal pressure changes, however, this was not considered in this paper as constant flow was studied. Yet, being able to include temporal changes is essential for studies on the cardiovascular system.

A drawback of the presented study is the large magnitude of the standard deviation. This is likely a consequence of the poor signal-to-noise ratio (SNR) that exists at the depth of $100 \mathrm{~mm}$ when only using five unfocused emission for creating an image. Increasing the number of firings per image would improve the SNR, but also reduce the effective frame-rate, which can be undesirable when studying the rapid flow of the heart. Furthermore, another shortcoming of the proposed method is its lacking ability to estimate the out-of-plane velocity component $v_{y}$. This makes the estimator vulnerable in complex flow environments that has vector velocity components in all three spatial direction.

Unlike other methods that use ultrasound flow data to derive the intravascular pressure, this method is based on 2-D angle-independent velocity fields captured in the entire image plane. This provides a wider information background for determining the pressure changes, and as the method is independent of the insonifying angle it facilitates a scan procedure that is less operator-dependent. The above described improvements may assist, and even exceed the need for invasive pressure catheters that otherwise remain a frequently used tool.

\section{CONCLUSION}

A non-invasive method for deriving pressure changes in a large field of view using ultrasound has been presented. Relative changes in pressure were derived using the Navier-Stokes equation for incompressible fluids. The vector velocities inserted into the equation were estimated using a directional synthetic aperture flow method yielding the two in-plane velocity components at a frame rate proportional to the pulse repetition frequency. The obtained

pressure gradients were evaluated by comparison to a finite element simulation model. The result of this showed a standard deviation of $32 \%$ and a bias low of $25 \%$.

\section{REFERENCES}

[1] Baim, D. S. and Grossman, W., [Grossman's cardiac catheterization, angiography, and intervention], Lippincott Williams \& Wilkins (2000).

[2] Donohue, T. J., Kern, M. J., Aguirre, F. V., Bach, R. G., Wolford, T., Bell, C. A., and Segal, J., "Assessing the hemodynamic significance of coronary artery stenosis: Analysis of translesional pressure-flow velocity relations in patients," J. Am. Coll. Cardiol. 22(2), 449-458 (1993).

[3] Tonino, P. A. L., Bruyne, B. D., Pijls, N. H. J., Siebert, U., Ikeno, F., vant Veer, M., Klauss, V., Manoharan, G., Engstrøm, T., Oldroyd, K. G., Lee, P. N. V., MacCarthy, P. A., and Fearon, W. F., "Fractional flow reserve versus angiography for guiding percutaneous coronary intervention," N. Engl. J. Med 360(3), 213224 (2009).

[4] Felix, W. R., Sige, B., Gibson, R. J., Williams, J., Popky, G. L., Edelstein, A. L., and Justin, J. R., "Pulsed Doppler ultrasound detection of flow disturbances in arteriosclerosis," J. Clin. Ultrasound 4(4), 275-282 (1976).

[5] Ophir, J., Céspedes, I., Ponnekanti, H., Yazdi, Y., and Li, X., "Elastography: A quantitative method for imaging the elasticity of biological tissues," Ultrason. Imaging 13(2), 111-134 (1991).

[6] Celermajer, D. S., Sorensen, K. E., Gooch, V. M., Miller, Sullivan, I. D., Lloyd, J. K., Deanfield, J. E., and Spiegelhalter, D. J., "Non-invasive detection of endothelial dysfunction in children and adults at risk of atherosclerosis," Lancet 340(8828), 1111-1115 (1992). 
[7] Giddens, D. P., Zarins, C. K., and Glagov, S., "The role of fluid mechanics in the localization and detection of atherosclerosis," J. Biomech. Eng. 115, 588-594 (1993).

[8] Bonow, R. O., Carabello, B., de leon, A. C., Edmunds, L. H., Fedderly, B. J., Freed, M. D., Gaasch, W. H., McKay, C. R., Nishimura, R. A., O'Gara, P. T., Gibbons, R. J., Russell, R. O., Ryan, T. J., and Smith, S. C., "ACC/AHA Guidelines for the management of patients with valvular heart disease," J. Am. Coll. Cardiol. 32(5), 1486-1582 (1998).

[9] Holen, J., Aaslid, R., and Landmark, K., "Determination of pressure gradient in mitral stenosis with a non-invasive ultrasound Doppler technique," Acta med. scand. 32, 455-460 (1976).

[10] Evans, D. H., McDicken, W. N., Skidmore, R., and Woodcock, J. P., [Doppler Ultrasound, Physics, Instrumentation, and Clinical Applications], John Wiley \& Sons, New York (1989).

[11] Ohtsuki, S. and Tanaka, M., "Doppler pressure field deduced from the Doppler velocity field in an observation plane in a fluid," Ultrasound Med. Biol. 29(10), 1431-1438 (2003).

[12] Olesen, J. B., Traberg, M. S., Pihl, M. J., and Jensen, J. A., "Noninvasive esimation of 2-D pressure gradients in steady flow using ultrasound," IEEE Trans. Ultrason., Ferroelec., Freq. Contr. 61(8), 1409-1418 (2014).

[13] Olesen, J. B., Villagomez-Hoyos, C. A., Traberg, M. S., and Jensen, J. A., "Non-invasive estimation of pressure changes along a streamline using vector velocity ultrasound," Proc. IEEE Ultrason. Symp. , 1-4 (2015).

[14] Prandtl, L., [Essentials of Fluid Dynamics], London: Blackie \& Son (1952).

[15] Wood, N. B., "Aspects of fluid dynamics applied to the large arteries," J. Theor. Biol. 199(953), 137-161 (1999).

[16] Jensen, J. A. and Nikolov, S. I., "Directional synthetic aperture flow imaging," IEEE Trans. Ultrason., Ferroelec., Freq. Contr. 51, 1107-1118 (2004).

[17] Jensen, J. A. and Oddershede, N., "Estimation of velocity vectors in synthetic aperture ultrasound imaging," IEEE Trans. Med. Imag. 25, 1637-1644 (2006).

[18] Lai, S. S. M., Yiu, B. Y. S., Poon, A. K. K., and Yu, A. C. H., "Design of anthropomorphic flow phantoms based on rapid prototyping of compliant vessel geometries," Ultrasound Med. Biol. 39(9), 1654-1664 (2013).

[19] Villagomez-Hoyos, C. A., Stuart, M. B., Bechsgaard, T., Nielsen, M. B., and Jensen, J. A., "High frame rate synthetic aperture vector flow imaging for transthoracic echocardiography," SPIE_U_SYMP (2016).

[20] Savitzky, A. and Golay, M. J. E., "Smoothing and differentiation of data by simplified least squares procedures," Anal. Chem. 36(8), 1627-1639 (1964). 